Acknowledgements: We would like to acknowledge the 13 medical consultants who assessed the films. We are grateful to L. Bradshaw (TB Section, Health Protection Agency Centre for Infections, London, UK) for proofreading the manuscript. No external funding was received for this study.

\section{REFERENCES}

1 Coker R, Bell A, Pitman R, et al. Tuberculosis screening in migrants in selected European countries shows wide disparities. Eur Respir J 2006; 27: 801-807.

2 Story A, Murad S, Verheyen M, et al. Tuberculosis in London - the importance of homelessness, problem drug use and prison. Thorax 2007; 62: 667-671.

3 World Health Organisation. Western Pacific Region. Assessing tuberculosis prevalence through population-based surveys. Geneva, World Health Organization, 2007.

4 Balabanova Y, Coker R, Fedorin I, et al. Variability in interpretation of chest radiographs among Russian clinicians and implications for screening programmes: observational study. BMJ 2005; 331: 379-382.

5 Shaw NJ, Hendry M, Eden OB. Inter-observer variation in interpretation of chest X-rays. Scott Med J 1990; 35: 140-141.

6 Nyboe J. Results of the international study on x-ray classification. Bull Int Union Tuberc 1968; 41: 115-124.

7 den Boon S, White NW, van Lill SW, et al. An evaluation of symptom and chest radiographic screening in tuberculosis prevalence surveys. Int J Tuberc Lung Dis 2006; 10: 876-882.

8 den Boon S, Bateman ED, Enarson DA, et al. Development and evaluation of a new chest radiograph reading and recording system for epidemiological surveys of tuberculosis and lung disease. Int J Tuberc Lung Dis 2005; 9: 1088-1096.

9 Graham S, Das GK, Hidvegi RJ, et al. Chest radiograph abnormalities associated with tuberculosis: reproducibility and yield of active cases. Int J Tuberc Lung Dis 2002; 6: 137-142.

10 Zellweger JP, Heinzer R, Touray $M$, et al. Intra-observer and overall agreement in the radiological assessment of tuberculosis. Int J Tuberc Lung Dis 2006; 10: 1123-1126.

11 de Vries G, van Hest RA, Richardus JH. Impact of mobile radiographic screening on tuberculosis among drug users and homeless persons. Am J Respir Crit Care Med 2007; 176: 201-207.

12 Crofts JP, Gelb D, Andrews N, et al. Investigating tuberculosis trends in England. Public Health 2008; 122: 1302-1310.

13 CDC. Instructions to panel physicians for completing chest $\mathrm{x}$-ray and classification worksheet (DS-3024). www.cdc.gov/ncidod/ dq/dsforms/3024.htm Date last updated: August 9, 2006. Date last accessed: June 4, 2008.

14 Nakamura K, Ohmi A, Kurihara T, et al. [Studies on the diagnostic value of $70 \mathrm{~mm}$ radiophotograms by mirror camera and the reading ability of physicians.] Kekkaku 1970; 45: 121-128.

15 Frieden T. Toman's Tuberculosis. Case Detection, Treatment and Monitoring. Geneva, World Health Organisation, 2004; pp. 92-95.

16 Dasgupta K, Menzies D. Cost-effectiveness of tuberculosis control strategies among immigrants and refugees. Eur Respir J 2005; 25 1107-1116.

17 Tulsky JP, Hahn JA, Long HL, et al. Can the poor adhere? Incentives for adherence to TB prevention in homeless adults. Int $J$ Tuberc Lung Dis 2004; 8: 83-91.

\title{
Importance of identifying Mycobacterium bovis as a causative agent of human tuberculosis
}

\section{To the Editors:}

The case study described hereafter emphasises the potentially vital importance of Mycobacterium bovis identification for appropriate tuberculosis patient management.

M. bovis, the classical causative agent of bovine tuberculosis, can be responsible for human TB, which makes this bacterium an important zoonotic species. In developed countries, the introduction of pasteurisation, preventing contamination from milk of infected cows, and eradication programmes for infected herds have considerably reduced the prevalence of human disease due to the bovine TB bacillus, but have not completely eradicated it [1-3]. Nevertheless, in many developed countries, the possibility of TB due to M. bovis infection, instead of Mycobacterium tuberculosis, is considered unlikely or even disregarded by microbiologists and clinicians.

Distinction of $M$. bovis from $M$. tuberculosis has significant relevance to patient management. In contrast to the other members of the $M$. tuberculosis complex, M. bovis is intrinsically resistant to pyrazinamide [4]. Beyond its use for specific $M$. bovis identification, this natural resistance is particularly important to consider. Pyrazinamide is usually given in the classical first-line TB treatment, as it is an effective sterilising drug that helps to shorten TB therapy due to its synergistic effect with rifampicin [5]. Thus, in case of M. bovis infection, pyrazinamide would be ineffective if implemented in a patient's anti-TB regimen. Unfortunately, results of in vitro susceptibility assays to pyrazinamide by conventional methods are not systematically correlated to in vivo activity. Susceptibility is, therefore, not often routinely assayed in laboratories. In addition, TB due to $M$. bovis is clinically, radiologically and microscopically indistinguishable from disease caused by $M$. tuberculosis. After culture on solid media, M. bovis displays specific characteristics like dysgonic colonies, and negative biochemical test results for nitrate reduction and niacin accumulation. However, clinical laboratories are increasingly using automated liquid culture systems followed by molecular tests for faster isolation and identification. Classical biochemical tests to identify $M$. bovis are not applicable using such liquid media, and most classical molecular identification methods based on targets like IS6110, 16S rDNA, 23S rDNA or ITS, do not distinguish between $M$. bovis and the other members of the $M$. tuberculosis complex. Other genetic markers [6] and the single commercial test (GenoType Mycobacterium, Hain, Nehren, Germany) allowing distinction between $M$. tuberculosis complex members are not widely used. 


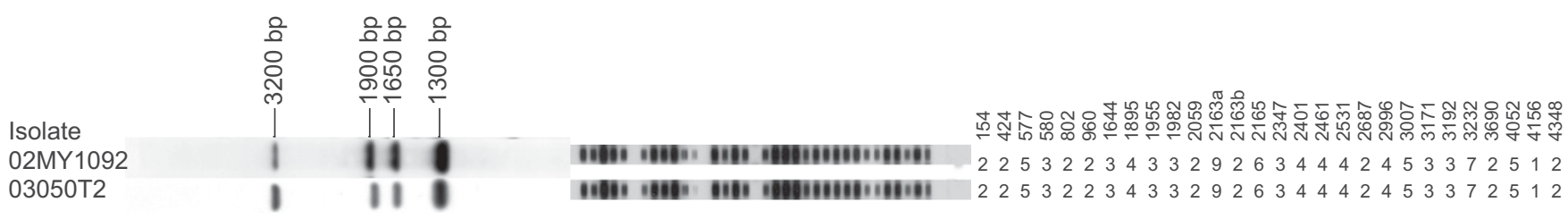

b)

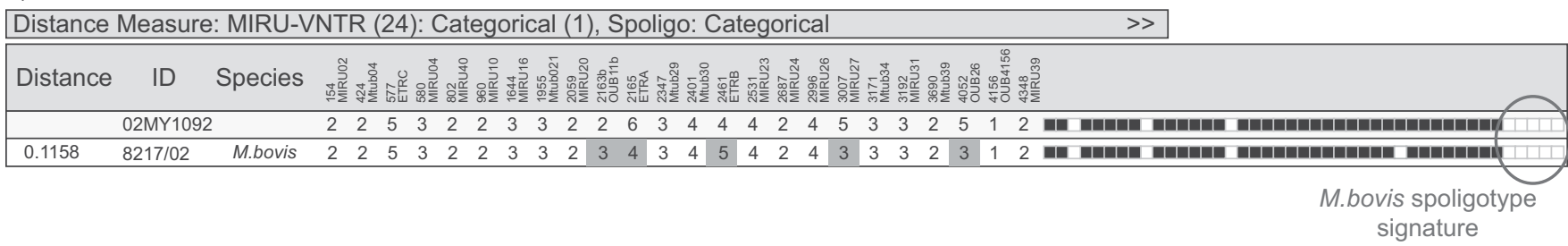

FIGURE 1. Mycobacterium bovis strain genotypes and identification. a) Comparison of human (above) and bovine (below) isolate genotypes. b) M. bovis identification based on MIRU-VNTRplus best match analysis. Grey-shaded boxes indicate variant mycobacterial interspersed repetitive unit variable-number tandem repeat (MIRU-VNTR) alleles between the human isolate and an M. bovis reference strain. Please note that identification in MIRU-VNTRplus is based on the set of 24 MIRU-VNTR loci standardised for Mycobacterium tuberculosis [8, 11]. According to standardisation, MIRU-VNTR markers are designated by their position in kbp on the M. tuberculosis H37Rv chromosome. RFLP: restriction fragment length polymorphism.

Here, we report a case of a 70-yr-old female living on a farm in Belgium. She was admitted in May 2001 with altered health condition and important weight loss, with a weight of only $32 \mathrm{~kg}$. TB was diagnosed, and considered to be a reactivation of an infection contracted previously, as pulmonary lesions were already documented in 1994. At the time of hospitalisation, her sputa were strongly smear positive and the mycobacteria obtained from culture were identified as $M$. tuberculosis complex using a routine in-house PCR test based on IS6110 insertion sequence. Susceptibility testing for three first-line drugs (isoniazid, rifampicin and ethambutol) was performed, and isolates were susceptible to these three drugs. The patient received a classical tritherapy comprising isoniazid, rifampicin and pyrazinamide (in Belgium, ethambutol is normally prescribed only for patients originating from high TB incidence countries). She did not tolerate per os medication and received intravenous regimen. However, she developed drug hepatitis and the dosage was adjusted. Cavitary lesions on chest radiography initially enlarged during hospitalisation and sputa became negative only 6 weeks later. The patient gained $4.5 \mathrm{~kg}$ of weight $(36.5 \mathrm{~kg})$ and left the hospital with the same regimen of antituberculous drugs, two months after her admission.

5 months later, in December 2001, the patient, who was still on standard isoniazid and rifampicin follow-up regimen, was readmitted with increasing cough and dyspnoea, and a weight of $34.5 \mathrm{~kg}$. There was wheezing on both lungs with a sort of lapping at the upper left lung lobe. Thorax radiography showed that this lobe was largely damaged and replaced by a large cavity. Although culture was negative after 4 weeks, sputum was smear positive.

During this second admission, transaminases suddenly increased, and gastroenterology examination suggested a novel episode of isoniazid-related hepatitis. This sudden recurrence of hepatitis was thought to result from lack of compliance of the patient at home and restored adherence to her anti-TB treatment after readmission. Therefore, isoniazid was replaced by pyrazinamide in addition to ethambutol in the follow-up regimen. The patient left the hospital after 3 weeks. She died from respiratory insufficiency 2 months later.

The mycobacterial strain infecting this patient was subsequently identified as $M$. bovis, independently from the above clinical history as follows. In 2002, the veterinary inspection service detected TB lesions on a cow at the slaughterhouse and subsequently a TB outbreak in cattle due to M. bovis on a farm. Epidemiological inquiry mentioned a case of human TB in the farmer's family, namely the patient described above. Consequently, the veterinary public health service alerted the medical public health service. Mycobacterial interspersed repetitive unit variable-number tandem repeat (MIRU-VNTR) typing based on 28 loci [7, 8], spoligotyping [9] and IS6110 restriction fragment length polymorphism [10] typing concordantly revealed fully matching genotypes for human and bovine isolates and disclosed, at the same time, the $M$. bovis identification of the patient's isolate (fig. 1) [11].

The two M. bovis isolates displayed a rare four-IS6110 band fingerprint observed in only $3 \%$ of Belgian herds [7]. This observation rendered even more likely the conclusion that both isolates represented infection by the same $M$. bovis strain, based on the concordant results of the three genotyping techniques. Although the genotype identity of the human and bovine isolates does suggest zoonotic infection acquired on this farm, a definitive scenario for the transmission route could not be established. No culture was available to confirm the patient's suspected previous TB episode dating back to 1994 and thus to confirm or exclude subsequent reactivation of such infection. Moreover, retrospective investigation of available veterinary inspection data did not allow us to establish when the TB outbreak precisely started in this herd. 
Regardless and more importantly, the initial absence of $M$. bovis identification of this patient's isolate compromised the efficiency of her treatments, and plausibly influenced the final fatal outcome. Because of this lack of specific identification, pyrazinamide was inadequately administered in the 2 months of induction chemotherapy and, worst, was inappropriately used to replace isoniazid in the follow-up treatment because of toxic hepatitis.

If the pneumologist had initially known that $M$. bovis was the causative agent, a treatment with isoniazid, rifampicin, and ethambutol, instead of pyrazinamide, would probably have been started. For the second episode, the pneumologist would probably have added a quinolone, again instead of pyrazinamide, in addition to ethambutol and rifampicin. In this context, nonutilisation of pyrazinamide would additionally have avoided hepatotoxic effects also known to be associated with this drug, for a patient prone to anti-TB drug-related hepatitis.

In conclusion, our report shows that absence of specific identification of $M$. bovis may have adverse consequences for TB patient management. We believe that even in high-income countries, human TB due to $M$. bovis is underestimated, because of frequent use of identification techniques that do not specifically distinguish $M$. bovis from the rest of the $M$. tuberculosis complex, and because susceptibility to pyrazinamide is not systematically tested. Therefore, clinical laboratories should routinely use molecular tests to differentiate $M$. bovis from $M$. tuberculosis and/or systematically check resistance to pyrazinamide. In addition, our report constitutes an additional example of the persistent significance of $M$. bovis as a zoonotic pathogen [1, 2], even in countries such as Belgium, which has been declared officially free of cattle TB according to the European Commission decision 2003/467/EC. Finally, it illustrates that molecular-guided cooperation between human and veterinary health services can improve detection of zoonoses.

C. Allix-Béguec ${ }^{*, \#, \uparrow_{1}, * *}$, M. Fauville-Dufaux ${ }^{*, * *}$, K. Stoffels, D. Ommeslag ${ }^{+}$, K. Walravens ${ }^{\S}$, C. Saegerman ${ }^{\dagger}$ and P. Supply $\#$, *Institut Scientifique de Santé Publique, Direction maladies infectieuses et transmissibles, ex Institut Pasteur, Brussels, ${ }^{+}$Pneumologie, AZ Sint-Lucas, Gent, ${ }^{\S}$ Centre d'Etude et de Recherches Vétérinaires et Agrochimiques, Brussels, and ${ }^{f}$ Research Unit of Epidemiology and Risk Analysis applied to Veterinary sciences (UREAR), Faculty of Veterinary Medicine, University of Liege, Liège, Belgium. "INSERM U629, and "Institut Pasteur de Lille, Lille, France. ${ }^{* *} \mathrm{C}$. Allix-Béguec and M. Fauville-Dufaux contributed equally to this work.

Correspondence: P. Supply, Institut de Biologie de Lille - 1, rue du Pr. Calmette, 59000 Lille Cédex, France. E-mail: philip. supply@ibl.fr

\section{SUPPORT STATEMENT}

C. Allix-Béguec was a fellow of the Brussels-Capital Region, Belgium and P. Supply is a researcher of the Centre National de la Recherche Scientifique (CNRS, Lille, France).

Statement of Interest: None declared.

Acknowledgements: P. Sonck (Institut Scientifique de Santé Publique, Direction maladies transmissibles et infectieuses, ex Institut Pasteur, Brussels, Belgium) is gratefully acknowledged for expertise in IS6110 restriction fragment length polymorphism analysis.

\section{REFERENCES}

1 Evans JT, Smith EG, Banerjee A, et al. Cluster of human tuberculosis caused by Mycobacterium bovis: evidence for personto-person transmission in the UK. Lancet 2007; 369: 1270-1276.

2 LoBue PA, Moser KS. Treatment of Mycobacterium bovis infected tuberculosis patients: San Diego County, California, United States, 1994-2003. Int J Tuberc Lung Dis 2005; 9: 333-338.

3 Rivero A, Marquez M, Santos J, et al. High rate of tuberculosis reinfection during a nosocomial outbreak of multidrug-resistant tuberculosis caused by Mycobacterium bovis strain B. Clin Infect Dis 2001; 32: 159-161.

4 O'Donohue WJ Jr, Bedi S, Bittner MJ, et al. Short-course chemotherapy for pulmonary infection due to Mycobacterium bovis. Arch Intern Med 1985; 145: 703-705.

5 Blumberg HM, Burman WJ, Chaisson RE, et al. American Thoracic Society/Centers for Disease Control and Prevention/Infectious Diseases Society of America: treatment of tuberculosis. Am J Respir Crit Care Med 2003; 167: 603-662.

6 Brosch R, Gordon SV, Marmiesse M, et al. A new evolutionary scenario for the Mycobacterium tuberculosis complex. Proc Natl Acad Sci USA 2002; 99: 3684-3689.

7 Allix C, Walravens K, Saegerman C, et al. Evaluation of the epidemiological relevance of variable-number tandem-repeat genotyping of Mycobacterium bovis and comparison of the method with IS6110 restriction fragment length polymorphism analysis and spoligotyping. J Clin Microbiol 2006; 44: 1951-1962.

8 Supply P, Allix C, Lesjean S, et al. Proposal for standardization of optimized mycobacterial interspersed repetitive unit-variablenumber tandem repeat typing of Mycobacterium tuberculosis. J Clin Microbiol 2006; 44: 4498-4510.

9 Kamerbeek J, Schouls L, Kolk A, et al. Simultaneous detection and strain differentiation of Mycobacterium tuberculosis for diagnosis and epidemiology. J Clin Microbiol 1997; 35: 907-914.

10 van Embden JD, Cave MD, Crawford JT, et al. Strain identification of Mycobacterium tuberculosis by DNA fingerprinting: recommendations for a standardized methodology. J Clin Microbiol 1993; 31: 406-409.

11 Allix-Beguec C, Harmsen D, Weniger T, et al. Evaluation and strategy for use of MIRU-VNTRplus, a multifunctional database for online analysis of genotyping data and phylogenetic identification of Mycobacterium tuberculosis complex isolates. J Clin Microbiol 2008; 46: 2692-2699. 